\title{
Immunothérapie et tube digestif Conclusion
}

\author{
X. Treton \\ (C) Lavoisier SAS 2015
}

A la lecture de ce dossier thématique on aura mesuré les énormes progrès réalisés en une dizaine d'années, dans le domaine de l'immunologie appliquée aux pathologies d'organes.

Ces progrès sont légèrement plus avancés en cancérologie que dans le domaine des MICI, pour l'instant. La caractérisation de l'infiltrat immunitaire et inflammatoire au contact des cellules tumorales, son évolution et son caractère prédictif, a changé la façon de percevoir le cancer colorectal. Grâce aux données de ce profil immunitaire, en le couplant au phénotype MSI/MSS, les patients pourront certainement très bientôt, recevoir un traitement personnalisé encore plus efficace. Même si elles ne sont pas encore validées, les thérapies immunologiques, basées sur une vaccination tumorale et/ou un transfert adoptif de cellules immunes autologues, viendront certainement bientôt enrichir l'arsenal thérapeutique.

Dans les MICI, probablement en raison de moyens de recherche plus limités, mais aussi de par leur facteurs physiopathologiques plus variés et inhomogènes, les avancées sont moindres. Cependant, la révolution thérapeutique connue lors de l'introduction des anti-TNF, dans la fin des années 1990, qui a été possible grâce à l'immunologie, n'a pas connu d'égal depuis. Ceci, en dépit d'une recherche acharnée, pour développer des biothérapies ciblant d'autres cytokines ou molécules régulatrices du système immunitaire.

Dans les MICI, il existe une discordance importante entre les observations "immunologiques » impliquées dans la physiopathologie et le fait de cibler spécifiquement ces voies, dans un but thérapeutique. Par exemple, si l'IL-17 est clairement impliquée dans les processus inflammatoires chroniques, les anticorps anti-IL-17 ont plutôt aggravé la maladie de Crohn des patients inclus dans les essais et favorisé les infections (majoritairement fungiques). Il n'existe à ce jour, à l'inverse de l'oncologie, aucun biomarqueur efficace dans les MICI permettant de définir le profil évolutif de la maladie, ou de prédire la réponse aux traitements. Par ailleurs, les essais basés sur une thérapie cellulaire sont plus limités.

Un essai de phase III évalue actuellement l'efficacité de lymphocytes $T$ régulateurs autologues, stimulés ex vivo par l'ovalbumine, dans la maladie de Crohn. D'autre part, plusieurs essais modestes, ont fourni des résultats encourageants, dans le traitement des fistules ano-périnéales crohniennes, par injection locale de cellules souches adipeuses autologues ou issues d'un donneur. Ces deux pistes nécessitent une confirmation, avant d'envisager leur développement.

Enfin, dans les formes sévères de maladie de Crohn, la greffe de moelle osseuse, a permis l'obtention de réponses prolongées, au prix d'une mortalité/morbidité difficilement acceptable. Cependant, il est important de regarder les progrès réalisés dans les deux disciplines de façon transversale. L'exemple de la voie CTLA-4 est illustratif. Lorsque l'on utilise un anticorps neutralisant, comme l'ipilimumab, dans le mélanome, on bloque l'inhibition de la co-stimulation lymphocytaire. On réactive ainsi des lymphocytes T cytotoxiques, afin de détruire les cellules tumorales. Ce faisant, on induit des colites sévères expérimentales, identifiant ainsi cette voie CTLA-4, comme majeure dans les MICI. En revanche, l'abatacept, molécule de fusion CTLA-4, qui bloque la co-stimulation lymphocytaire, et améliore la colite de plusieurs modèles murins, est inefficace dans les MICI, voire aggrave la $\mathrm{RCH}$.

De quoi rester humble face à la complexité et la plasticité du système immunitaire, surtout lorsque l'on cherche à le moduler.

Les résultats des interventions thérapeutiques sont parfois inattendus, ou contre-intuitifs, mais restent une source majeure de progrès pour les années à venir. 